\title{
Chapter 5 \\ Polarity Control of Spindle Positioning in the C. elegans Embryo
}

\author{
Lars-Eric Fielmich and Sander van den Heuvel
}

\begin{abstract}
Cell and tissue polarity guides a large variety of developmental processes, including the choice between symmetric and asymmetric cell division. Asymmetric divisions create cell diversity and are needed for the maintenance of tissue-specific stem cells. Symmetric divisions, on the other hand, promote exponential cell proliferation. Polarized cells often divide symmetrically by cleaving along the axis of polarity. Alternatively, cell cleavage in a plane perpendicular to the polarity axis results in asymmetric division. To control this decision, developmental cues position the mitotic spindle, which instructs the plane of cell cleavage. In animal cells, the positioning of the spindle depends on evolutionarily conserved interactions between a heterotrimeric G-protein alpha subunit, TPR-GoLoco domain protein, and NuMA-related coiled-coil protein. This trimeric complex recruits the dynein microtubule motor and captures astral microtubules at the cortex. The interplay between dynein movement and depolymerizing microtubules generates cortical pulling forces that promote aster movement and spindle positioning. Through mechanisms that are poorly understood, cell polarity and other developmental signals control the microtubule-pulling forces to instruct the orientation and plane of cell division. In this chapter, we review the current understanding of the connection between cell polarity and spindle positioning, with a focus on studies of the early $C$. elegans embryo. The nematode $C$. elegans develops through a highly reproducible division pattern and has proven to be a powerful model for studying the regulation and execution of asymmetric cell division.
\end{abstract}

Keywords Asymmetric cell division $\bullet$ C. elegans $•$ Polarity $\bullet$ Pulling forces • Spindle positioning

\author{
Abbreviations \\ AB Anterior blastomere \\ EMS Endomesodermal blastomere
}

\footnotetext{
L. Fielmich • S. van den Heuvel (ه)

Developmental Biology, Utrecht University, Kruytbuilding O507, Padualaan 8,

$3584 \mathrm{CH}$ Utrecht, The Netherlands

e-mail: s.j.l.vandenheuvel@uu.nl
} 


$\begin{array}{ll}\text { GAP } & \text { GTPase-activating protein } \\ \text { GEF } & \text { Guanine nucleotide exchange factor } \\ \text { LIN } & \text { Lineage abnormal } \\ \text { NB } & \text { Neuroblast } \\ \text { P1 } & \text { Posterior blastomere 1 } \\ \text { PAR } & \text { Partitioning defective } \\ \text { PCP } & \text { Planar cell polarity } \\ \text { SOP } & \text { Sensory organ precursor }\end{array}$

\subsection{Spindle Positioning and Asymmetric Cell Division}

When cells divide, chromosome segregation is followed by cleavage of the cytoplasm. The microtubule spindle apparatus instructs the cytokinetic furrow to form perpendicular to, and usually midway through, the central spindle. By positioning the spindle with respect to the polarity axis of the cell or tissue, daughter cells are formed at the proper place, with the right size and developmental fate. Hence, accurate spindle positioning is critical for tissue integrity, morphogenesis, and the balance between symmetric and asymmetric division of stem cells and tissuespecific progenitor cells. How cell polarity information is translated into proper spindle positioning has been a subject of intense study over the past 15 years. Information from a variety of systems has resulted in a general model for spindle positioning in animal cells (for reviews: Galli and van den Heuvel 2008; Knoblich 2010; Morin and Bellaïche 2011). While some aspects are understood in considerable detail, cell type-dependent variations are still emerging, and many questions remain unanswered even for the best-studied systems.

The distinction is often made between intrinsic and extrinsic asymmetric division (Horvitz and Herskowitz 1992) (Fig. 5.1). In intrinsic asymmetric division, anterior-posterior, apical-basal, or planar polarity guides the asymmetric distribution of cell fate determinants in mitosis. By also aligning the mitotic spindle with this polarity axis, cytoplasmic cleavage segregates the localized components into a single daughter cell. Thus, intrinsic asymmetric division generates different daughter cells during the cell division process. As an alternative mode of asymmetric division, external signals may instruct a different fate in daughter cells that are initially identical after division. As an example, tissue-specific stem cells may depend on contact with a niche for the maintenance of the uncommitted state. If the spindle orients perpendicular to the niche during cell division, a single daughter cell will remain associated with the niche as an uncommitted stem cell, while the other daughter cell loses this interaction and initiates a differentiation program (Fig. 5.1). Thus, spindle positioning is crucial for the unequal partitioning of determinants during intrinsic asymmetric division and for properly positioning daughter cells during extrinsic asymmetric division.

Spindle positioning has been best studied in the context of asymmetric cell division in invertebrate models. While this review focuses on the nematode 

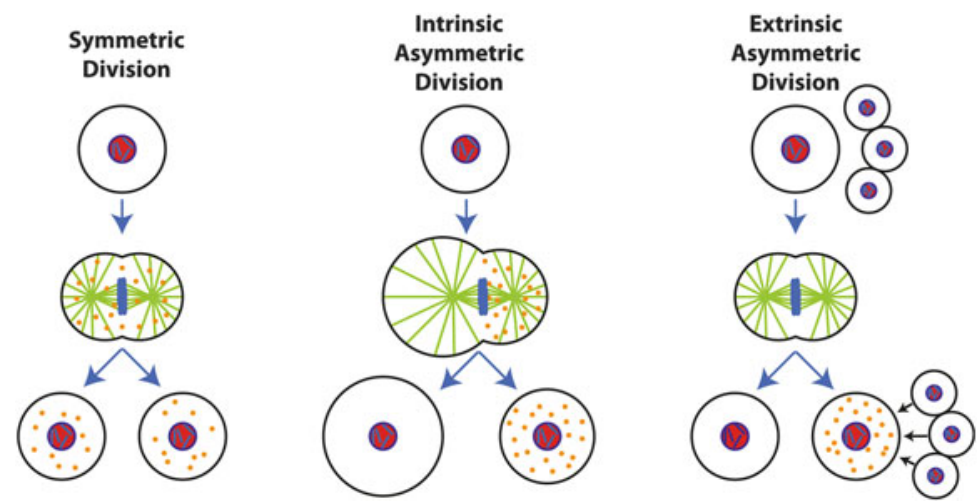

Fig. 5.1 Symmetric and asymmetric cell division. The left panel illustrates a symmetric cell division in which cell fate determinants (orange) are distributed equally over the two daughter cells. In the middle panel, an intrinsic asymmetric cell division is depicted. The plane of the cell cleavage and asymmetric localization of fate determinants in the mitotic mother cell ensure that cell division creates daughter cells with different cytoplasmic determinants and cell fates. Off-center positioning of the spindle causes the division to be asymmetric in size as well. The final panel illustrates an extrinsic asymmetric division. The two daughter cells do not inherit different fate determinants during mitosis, but receive different extrinsic signals that promote their distinct cell fates

C. elegans, other examples should be mentioned to illustrate the importance of this process. Excellent examples are the Drosophila central nervous system and larval brain, which are formed through repetitive rounds of intrinsic asymmetric division of neuroblasts (NBs). The embryonic neuroblasts become specified within a polarized epithelium known as ventral neuroectoderm (Knoblich 2008, 2010; Morin and Bellaïche 2011). They delaminate from this epithelium and go through several rounds of asymmetric divisions in which the spindle aligns along the apical-basal polarity axis. Critical in spindle orientation is the expression of the Inscuteable adaptor protein upon NB specification. Inscuteable forms a link between proteins that determine apical cell polarity and proteins that anchor astral microtubules, thereby ensuring apical-basal orientation of the spindle. The apical daughter cells are larger and retain self-renewing capacity, while the smaller basal cells (known as ganglion mother cells) undergo one symmetric division to form two neurons. Remarkably, the size asymmetry does not follow from asymmetric spindle positioning, but from asymmetry in spindle geometry during anaphase (Kaltschmidt et al. 2000). Consequently, the cell cleavage plane is placed toward the basal side. Asymmetric cell cleavage in neuroblasts can also occur independently of the spindle, presumably induced by basally enriched actomyosin (Cabernard et al. 2010).

Another well-studied model for asymmetric division is the Drosophila sensory organ precursor (SOP, also called pI) cell. SOP cells generate the mechanosensory organs of the peripheral nervous system of the fly. These organs consist of a sensory hair, connected to a socket cell and neuron, which is surrounded by a glial-like sheath cell (Knoblich 2008; Morin and Bellaïche 2011). To form these four 
different cells, SOP cells go through two rounds of intrinsic asymmetric cell division. These divisions are coordinated with the anterior-posterior (A/P) body axis of the fly to align the orientation of sensory bristles. Hereto, Frizzled (Fz)dependent planar cell polarity (PCP) signaling aligns the spindle along the A/P body axis in mitotic SOP cells. During this division, the Notch-antagonist Numb localizes to the anterior cell cortex and becomes asymmetrically segregated to the anterior pIIb cell. This cell continues to divide to form a neuron and sheath cell, while division of the posterior pIIa cell creates the hair and socket cell of the sensory organ.

Stem cell divisions in the male and female Drosophila germ line provide examples of niche-dependent asymmetric divisions. The mechanisms that control these divisions differ from the focus of this chapter and are therefore not discussed. Insight obtained in C. elegans studies has substantially contributed to the molecular understanding of asymmetric divisions in mammals, in particular those that occur during mammalian skin and brain development. The development of the mouse skin from a single to multilayered structure (stratification) coincides with the switch from symmetric to mostly asymmetric divisions around day 14 of embryogenesis (E14) (Lechler and Fuchs 2005; Williams et al. 2011). The spindle orients in the plane of the epithelium during symmetric divisions. Asymmetric division involves the rotation of the spindle to align with the apical-basal axis of cell polarity and leads to the formation of a differentiating daughter cell above the basal cell layer. The mammalian brain develops from neuroepithelial progenitor cells (Fietz and Huttner 2011). These cells are connected by adherens junctions close to their apical surface. Cell cycle-dependent apical-basal movements of the cell nuclei create a pseudostratified epithelium. When these cells enter mitosis, the nucleus is always at the apical side. Initially, the mitotic spindle orients within the plane of the epithelium, and symmetric divisions expand the pool of progenitors. Similar to skin development, a switch to asymmetric division leads to neurogenesis, which peaks around day E14-E15 of mouse embryogenesis and coincides with subtle spindle rotations. Because the apical surface is narrow, even a subtle spindle rotation leads to the creation of a daughter cell that lacks apical surface and adherens junction attachment (Fietz and Huttner 2011). This cell initiates neural differentiation either directly or after further division. In all these examples, apical polarity and spindle positioning use molecular mechanisms that have been discovered in substantial part through studies of the early $C$. elegans embryo.

\subsection{Spindle Positioning in the Early C. elegans Embryo}

The nematode $C$. elegans develops through a highly reproducible pattern of asymmetric and symmetric divisions. The division of the $C$. elegans zygote has served as a particularly informative model for the concerted steps that are required for intrinsic asymmetric cell division: establishment of polarity, asymmetric localization of fate determinants, and proper positioning of the spindle to instruct the plane 
of cell cleavage. The $C$. elegans oocyte is not polarized before fertilization. Sperm entry initiates a symmetry-breaking event, which defines the posterior end and leads to the formation of the embryonic $\mathrm{A} / \mathrm{P}$ axis. The establishment of $\mathrm{A} / \mathrm{P}$ polarity is excellently reviewed in another book chapter (Carrie Cowan, Volume 2). In short, the anterior PAR protein complex consists of the PDZ-domain proteins PAR-3 and PAR-6 in association with atypical protein kinase C (PKC-3). This complex occupies the oocyte cortex at the time of fertilization (Fig. 5.2). Two posterior PAR proteins, the PAR-2 ring finger protein and PAR-1 MARK family Ser/Thr kinase, are present in the cytoplasm at that time, because PKC-3 phosphorylates PAR-2 and prevents its cortical localization. While this distribution is stable, fertilization-dependent processes disturb the equilibrium. A sperm-derived Rho-GAP, CYK-4, and cortical depletion of the Rho-GEF ECT-2 by maturated sperm-derived centrosomes disrupt the actomyosin cytoskeleton and cause it to retract toward the opposite (anterior) pole. Coincident with this actomyosin flow, anterior PAR proteins are removed from the posterior cortex. Moreover, microtubules nucleated at the mature centrosomes bind PAR-2 and protect it from PKC-3 phosphorylation. This allows PAR-2 to occupy the cortex and to recruit PAR-1 near the paternal pronucleus (Fig. 5.2). PAR-1 then phosphorylates PAR-3, which antagonizes posterior localization of the anterior PAR complex. The mutual antagonism between the anterior and posterior PAR proteins results in a new equilibrium

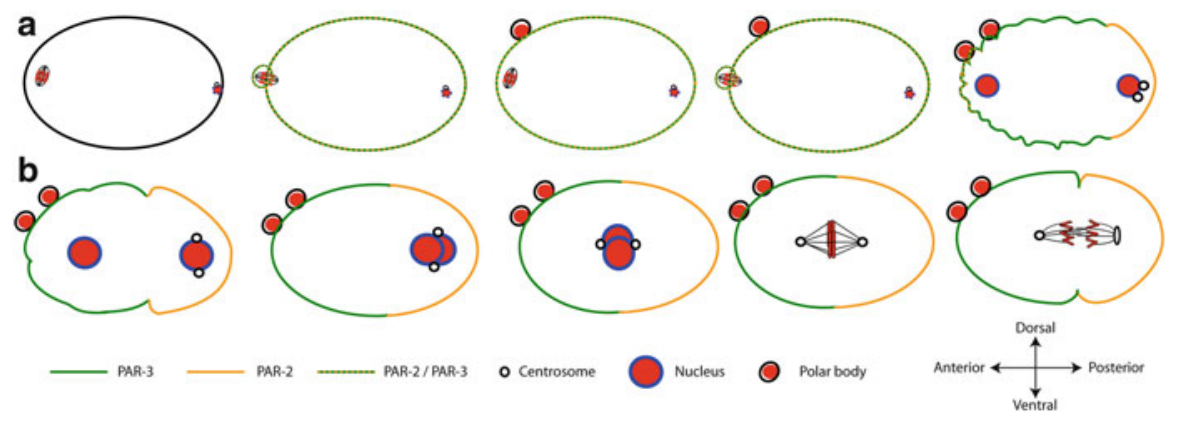

Fig. 5.2 Establishment of polarity in the C. elegans zygote. (a) At the moment of fertilization by the male sperm, the oocyte is stalled in prophase of meiosis I, and PAR polarity proteins are distributed uniformly. Sperm entry breaks the symmetry and marks the future posterior pole of the embryo. The oocyte then finishes meiosis I and II, resulting in two polar bodies and one maternal pronucleus. Simultaneously, cortical actomyosin starts to retract anteriorly, while the posterior cortex smoothens. Coincident with the actin flow, anterior PAR proteins (green) are removed from the posterior cortex, allowing for cortical localization of posterior PAR proteins (yellow). (b) Polarization is complete when an equilibrium between the opposing PAR domains is reached. After the pronuclei have met in the posterior, the pronuclei-centrosomal complex centrates and rotates while assembling the mitotic spindle along the A/P PAR polarity axis. At the embryonic midplane, the nuclear envelopes break down and chromosomes become aligned at the metaphase plate. Higher posterior pulling forces acting on the spindle cause the spindle to displace posteriorly, positioning the cleavage plane off-center. This sequence of events, initiated by male sperm entry, results in a division that is unequal in size and contents. Figure adapted after Galli and van den Heuvel (2008) 
with PAR-3/PAR-6/PKC-3 occupying the anterior half of the cortex, while PAR-2 and PAR-1 occupy the posterior half (Cowan and Hyman 2007; Hoege and Hyman 2013).

Polarity establishment coincides with two consecutive highly asymmetric meiotic divisions. These divisions produce two small polar bodies, as the compact meiotic spindle segregates the chromosomes in close proximity to the cortex (Fig. 5.2). Subsequently, the haploid maternal pronucleus migrates to the posterior to meet the paternal pronucleus, followed by the movement of the adjoined nuclei toward the center (centration). The pronuclei-centrosomal complex rotates during this anterior migration, coincident with spindle assembly along the $\mathrm{A} / \mathrm{P}$ axis of the zygote ( $\mathrm{P} 0$ ). Following nuclear envelope breakdown, the spindle aligns the chromosomes at the metaphase plate in the middle of the zygote. The spindle relocates slightly toward the posterior in metaphase and during elongation in anaphase. During this translocation, the posterior pole shows extensive lateral oscillations, named "rocking," while the anterior pole remains relatively fixed. The off-center placement of the spindle results in an unequal first division that gives rise to a larger anterior blastomere (AB) and smaller posterior daughter (P1) (Figs. 5.2 and 5.3). Coincidently, cytoplasmic determinants become unequally partitioned, creating intrinsic differences between the $\mathrm{AB}$ somatic blastomere and $\mathrm{P} 1$ germ line precursor cell. All these asymmetries depend on A/P polarity. In embryos that lack PAR protein function, the spindle remains in the center of the zygote, and cell cleavage is symmetric in size and fate. Inactivation of anterior PAR complex function results in the rocking of both spindle poles and exaggerated spindle elongation (Kemphues et al. 1988). In contrast, inactivation of posterior PAR function causes both spindle poles to remain quite stationary, resembling the normal behavior of the anterior pole.

The P1 blastomere reestablishes opposing PAR protein domains. The duplicated centrosomes migrate around the nucleus in $\mathrm{AB}$ and $\mathrm{P} 1$ to initiate a transverse spindle position (Fig. 5.3). In P1, however, the nucleus and associated centrosomes rotate by $90^{\circ}$ to align with the $\mathrm{A} / \mathrm{P}$ polarity axis in prometaphase and promote asymmetric cell division (Fig. 5.3). This division generates another precursor of the germ line (P2), which continues cell-autonomously controlled asymmetric division, just like its daughter germ line precursor cell P3. In contrast, asymmetric division of EMS, a precursor of endoderm (intestine) and mesoderm, requires signaling from the neighboring P2 blastomere at the four-cell stage. This involves a Wnt/Fz pathway and parallel acting MES-1/SRC-1 tyrosine kinase signaling. These pathways coordinate spindle orientation along the long axis of the embryo with endoderm specification in the daughter cell that contacts P2 (Bei et al. 2002). The division of $\mathrm{ABa}$ and $\mathrm{ABp}$, the other two blastomeres of the four-cell embryo, uses a small rotation of the spindle to divide left-right under a slight angle to create reproducible left-right asymmetry of the animal (Bergmann et al. 2003). The right daughter cell of this division, ABar, rotates its spindle again dependent on a Wnt-signal from the neighboring $\mathrm{C}$ blastomere. In summary, the position of the 


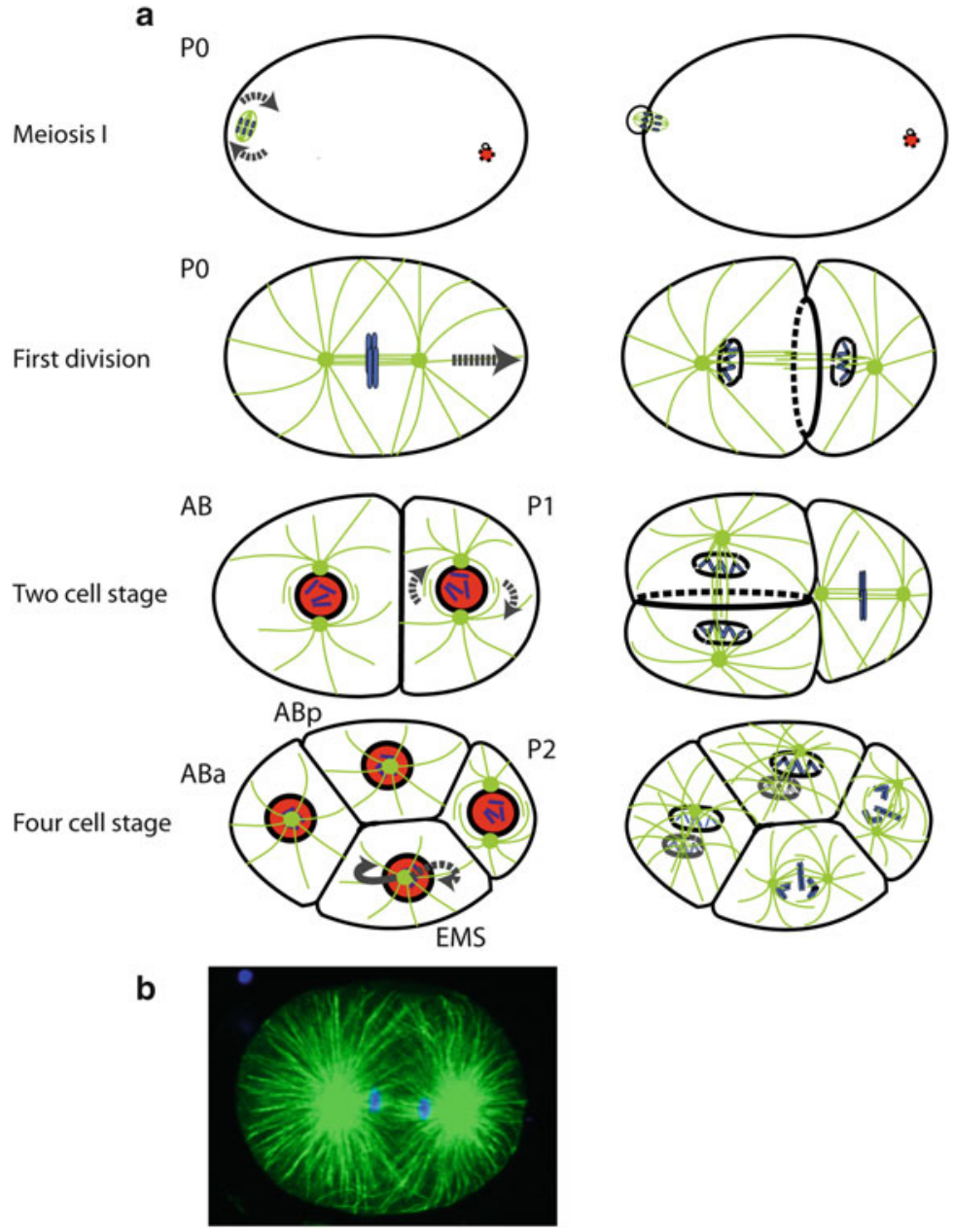

Fig. 5.3 Asymmetric cell divisions in the C. elegans early embryo. (a) During meiosis, the oocyte divides highly asymmetrically because the meiotic spindle locates close to the cell cortex and rotates such that small polar bodies are formed, and a large zygote remains. During the first mitotic division, pulling forces are asymmetric in $\mathrm{P} 0$, resulting in a larger $\mathrm{AB}$ and smaller $\mathrm{P} 1$ blastomere. In $\mathrm{P} 1$, the spindle rotates and aligns with the $\mathrm{A} / \mathrm{P}$ polarity axis as in $\mathrm{P} 0$. In the four-cell embryo, $\mathrm{ABa}$ and $\mathrm{ABp}$ divide under a slight angle to generate left-right asymmetry. The EMS spindle rotates under the influence of extrinsic signals emanating from the P2 cell. These rounds of asymmetric division are highly reproducible and regulated, making them a strong experimental model for studies of asymmetric cell division. (b) Immunofluorescent labeling of tubulin (green) and DNA (blue) marks the mitotic spindle, which is visibly shifted posteriorly during P0 division. Figure adapted after Galli and van den Heuvel (2008)

spindle and cleavage plane is highly regulated in C. elegans and critical in generating the proper cell lineages throughout development. While substantial insight has been obtained in the proteins that contribute to spindle positioning, the coordination with cell polarity is only partly understood. 


\subsection{The Molecular Components of Cortical Force Generation}

In animal cells, pulling forces that act between the cell cortex and astral microtubules position the spindle in mitosis. These forces are generated by depolymerization of the microtubule plus ends, in association with dynein minus-end-directed motor proteins. The dynein motor is recruited to the cortex by a trimeric protein complex that is conserved throughout the animal kingdom. In $C$. elegans, the complex contains a GOA-1 or GPA-16 heterotrimeric G-protein $\alpha$-subunit, which functions as a cortical anchor. The TPR and "G-protein regulator" motif proteins GPR-1/GPR-2 form a bridge between $\mathrm{G} \alpha$ at the membrane and the LIN-5 coiledcoil protein. LIN-5 interacts directly or indirectly with subunits of the cytoplasmic dynein complex (Fig. 5.4). Genetic studies place PAR polarity proteins upstream of G $\alpha$-GPR-1/GPR-2-LIN-5 in the control of spindle positioning. Multiple possible links between polarity regulators and the pulling force complex have been suggested, but a comprehensive picture of spatiotemporal control of spindle positioning has yet to emerge.

\subsubsection{Noncanonical G-Protein Signaling}

G-protein signaling has long been known as a major route to convey extracellular signals over the plasma membrane. In this pathway, ligand binding induces a

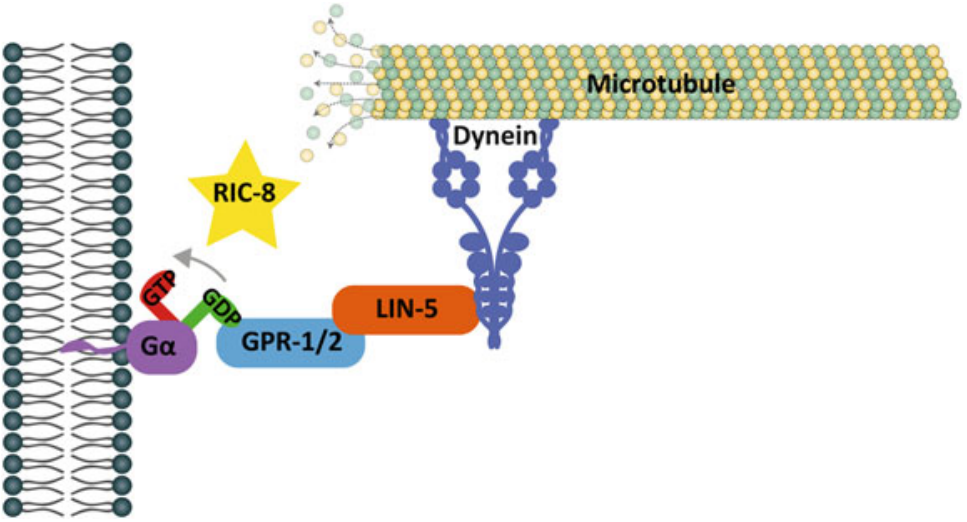

Fig. 5.4 Model illustrating the generation of cortical pulling forces at microtubule plus ends. Dynein is tethered to the cortex by a trimeric complex of Go.GDP-GPR-1/GPR-2-LIN-5. Cortical pulling forces are generated by microtubule depolymerization and dynein minus-end-directed motor activity. The GEF protein RIC-8 facilitates the GDP/GTP exchange on GOA-1 G $\alpha$ and promotes GPA-16 G $\alpha$ plasma membrane localization. Inactivation of any of these components abrogates cortical pulling force generation. See text for further explanation 
transmembrane G-protein-coupled receptor (GPCR) to act as a guanine nucleotide exchange factor (GEF). The exchange of GDP to GTP causes dissociation of the receptor-associated G $\alpha \cdot G D P-G \beta \gamma$ complex, allowing G $\alpha \cdot G T P$ and G $\beta \gamma$ to activate downstream targets. Surprisingly, spindle positioning turned out to use a receptorindependent noncanonical G-protein pathway. The first support for heterotrimeric G-protein contribution to spindle positioning came from the Plasterk group, who found that inactivation of $g p b-1$, one of two $C$. elegans $\mathrm{G} \beta$ genes, randomizes mitotic spindle orientation in early divisions and thus perturbs the tightly regulated process of asymmetric cell division (Zwaal et al. 1996). Subsequently, Miller and Rand found that mutation of goa- 1 Goo also affects the position and orientation of the mitotic spindle in early C. elegans embryos (Miller and Rand 2000). While in goa-1 mutant embryos defects were observed with low penetrance, combining mutations and/or RNA-mediated interference (RNAi) of goa- 1 and gpa-16 Goi/o causes near-complete absence of spindle positioning and results in symmetric division of the one-cell embryo (Gotta and Ahringer 2001). Thus, the GOA-1 and GPA-16 G $\alpha$ subunits (together referred to as $\mathrm{G} \alpha$ ) act redundantly in spindle positioning. Finally, RNAi of one of two C. elegans $\mathrm{G} \gamma$-subunit genes, gpc-2 $(\mathrm{G} \gamma-2)$, was found to cause spindle defects comparable to gpb-1 RNAi (Gotta and Ahringer 2001). At that time, a complete heterotrimeric $\mathrm{G}$ protein, consisting of GOA-1/GPA-16 G $\alpha$, GPB-1 G $\beta$, and GPC-2 G $\gamma$ subunits, had been implicated in asymmetric cell division in $C$. elegans. There was no evidence, however, for the contribution of a G-protein-coupled receptor (GPCR), and transmembrane signaling appeared unlikely in a one-cell embryo.

Support for noncanonical G-protein signaling in asymmetric cell division came simultaneously from studies in Drosophila. Inscuteable was found to control asymmetric NB divisions in association with the Partner of Inscuteable (Pins) protein and a $\mathrm{G} \alpha$ subunit (Schaefer et al. 2000). In addition, Goi and Pins, but not Inscuteable, were shown to determine the division orientation of SOP cells in Drosophila (Bellaïche et al. 2001; Schaefer et al. 2001). Thus, cell autonomous control of spindle positioning in the C. elegans zygote and Drosophila NB and division orientation control by planar cell polarity all turned out to use a novel form of G-protein signaling.

\subsubsection{TPR-GPR Domain Proteins}

Three groups simultaneously identified additional positive regulators of $\mathrm{G} \alpha$ in the control of asymmetric division of the $C$. elegans zygote (Colombo et al. 2003; Gotta et al. 2003; Srinivasan et al. 2003). The G-protein regulator (GPR) genes gpr-1 and gpr-2 were first observed to affect spindle positioning in a high-throughput RNAi screen of all chromosome III-encoded genes (F22B7.13, C38C10.4; (Colombo et al. 2003)). Independently, GPR-1 and GPR-2 proteins were co-immunopurified with the spindle-positioning protein LIN-5 (Srinivasan et al. 2003). The gpr-1 and gpr-2 coding sequences share $96 \%$ nucleotide identity; hence, RNAi for one 
inhibits the other gene simultaneously. The predicted proteins are $97 \%$ identical and are commonly referred to together as GPR-1/GPR-2 or simply GPR. Importantly, GPR-1 and GPR-2 are related to Drosophila Pins and part of a protein family that includes the closely related mammalian LGN (Leu-Gly-Asn repeat-enriched protein) and AGS3 (activator of G-protein signaling), as well as C. elegans AGS-3. These proteins all contain multiple N-terminal tetratricopeptide (TPR) protein interaction motifs and at least one C-terminal GoLoco/GPR domain (Colombo et al. 2003; Gotta et al. 2003; Srinivasan et al. 2003).

The GoLoco/GPR domain interacts with Gai/o-GDP, inhibits GDP release, and competes with $\mathrm{G} \beta \gamma$ association. Crystal structure studies showed that $\mathrm{G} \beta \gamma$ and the GoLoco/GPR motif interact with G $\alpha \cdot$ GDP through overlapping binding sites (Kimple et al. 2002; Martin-mccaffrey et al. 2005). Hence, spindle positioning was initially thought to depend on G $\beta \gamma$ release (Gotta and Ahringer 2001; Schaefer et al. 2001). In C. elegans, RNAi of gpr-1/gpr-2 closely mimics goa-1/gpa-16 RNAi, which indicates that GPR-1 and GPR-2 act positively with Go. In contrast, gpb-1 G $\beta$ and gpc-2 G $\gamma$ RNAi do not resemble gpr-1/gpr-2 RNAi or alter the goa-1/ gpa-16 RNAi phenotype. Such observations demonstrated that, rather than G $\beta \gamma$, the interaction between $\mathrm{G} \alpha \cdot \mathrm{GDP}$ and GPR-1/GPR-2 is required for spindle positioning in asymmetric cell division (Colombo et al. 2003; Gotta et al. 2003; Srinivasan et al. 2003). This confirmed alternative use of G-protein signaling in spindle

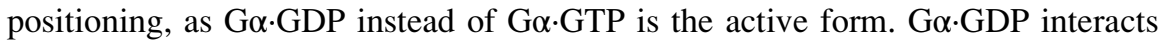
with a GPR/Pins family member, which in turn uses the TPR motifs, and possibly a linker between the TPR and GoLoco/GPR domains, to recruit additional spindlepositioning proteins to the membrane.

\subsubsection{LIN-5 (Mud/NuMA)}

Another important component of the cortical pulling force complex in C. elegans is the LIN-5 protein, which is the functional equivalent of mammalian NuMA (nuclear mitotic apparatus) and Drosophila Mud (mushroom body defect). The lin-5 gene was defined by "lineage-abnormal" mutations (Albertson 1978). Homozygous lin-5 mutants are sterile, but when derived from heterozygous parents complete normal embryonic development. Postembryonic cell divisions fail in mitosis in these mutants; chromosomes may not fully align at the metaphase plate; anaphase and cytokinesis do not occur, yet cells exit from mitosis at the normal time and enter the next round of DNA synthesis, centrosome duplication, and mitotic entry (Albertson 1978; Sulston and Horvitz 1981; Lorson et al. 2000). Dependent on the lineage, postembryonic blast cells in lin-5 mutants continue abortive mitoses and become highly polyploid. Embryonic development in these mutants is driven by maternal product, as knockdown of lin-5 by RNAi and temperature shift of lin-5(ev57lts) mutants cause complete embryonic lethality (Lorson et al. 2000).

The extended central domain of the LIN-5 protein is predicted to form an $\alpha$-helical coiled-coil structure (Lorson et al. 2000). While the amino acid sequence 
provided little functional information, immunopurification followed by mass spectrometry revealed that LIN-5 and GPR-1/GPR-2 form part of a protein complex (Srinivasan et al. 2003). The strong overlap in phenotype also supports joint functions: $g p r-1 / g p r-2$ and lin-5 RNAi each causes reduced spindle elongation, lack of posterior movement of the spindle in anaphase, and failure to undergo normal asymmetric division of the zygote (Gotta et al. 2003; Srinivasan et al. 2003). The characteristic oscillations and flattening of the posterior spindle pole that normally occur during spindle migration are also absent after gpr-1/gpr-2 and lin5 knockdown, and the spindle does not rotate in the P1 blastomere. Chromosome segregation and cytokinesis continue for two or three rounds of cell division, giving rise to dead embryos with a few highly polyploid nuclei. The physical association between LIN-5 and GPR-1/GPR-2, as well as interaction between GPR-1/GPR-2 and $\mathrm{G} \alpha$, and the strong resemblance in lin-5, gpr-1/gpr-2, and goa-1/gpa-16 RNAi phenotypes all supported a model in which the encoded proteins act together to control the mitotic spindle position. In addition, $\operatorname{lin}-5$ is also required for meiotic spindle rotation, independently of gpr-1/gpr-2 and goa-1/gpa-16 $\mathrm{G} \alpha$ (Lorson et al. 2000; van der Voet et al. 2009).

\subsubsection{The Trimeric Go-GPR-LIN-5 Complex Recruits Dynein to the Cortex}

The subcellular localizations of LIN-5 and GPR-1/GPR-2 show strong overlap and mutual dependence. The proteins are present at the spindle poles, in the cytoplasm, at the cell cortex, and, specifically in metaphase, at kinetochore microtubules. GPR-1/GPR-2 fails to localize in the absence of LIN-5, and LIN-5 loses its cortical localization when GPR-1/GPR-2 or G $\alpha$ is gone (Lorson et al. 2000; Srinivasan et al. 2003; van der Voet et al. 2009). The combined data support that Goi/o.GDP, GPR-1/GPR-2, and LIN-5 form a trimeric complex needed for spindle positioning in C. elegans (Fig. 5.4). Similarly, Drosophila Pins associates with Goi and the LIN-5/NuMA-related protein Mud in NBs and epithelial cells (Izumi et al. 2004; Bowman et al. 2006; Siller and Doe 2009). Moreover, mammalian LGN recruits the NuMA protein to the cell cortex and simultaneously interacts with Goi (Du and Macara 2004). Each of these complexes is critical for spindle positioning and orienting cell division, in mammals in particular in the developing skin and brain (Lechler and Fuchs 2005; Williams et al. 2011). Thus, an evolutionarily conserved mechanism appears to control the positioning of the cell division plane in all animals.

Observations in the one-cell C. elegans embryo provided additional functional insights. It was found that the Go-GPR-LIN-5 complex is needed for the generation of pulling forces that act from the cortex at astral microtubules. Such external forces can be made visible by means of spindle midzone severing with a UV laser (Grill et al. 2001). Following spindle severing, the spindle poles move outward with 
increased speed toward the cell periphery. Importantly, the posterior pole moves with a higher velocity and over a larger distance than the anterior pole. This indicates asymmetry in pulling forces, which depends on A/P polarity; par-2 mutant embryos are "anteriorized" and show anterior and posterior pole movements with the same low peak velocity as the wild-type anterior pole. Vice versa, par-3 mutant embryos are "posteriorized" with both sides showing high pulling forces (Grill et al. 2001). The knockdown of Go, gpr-1/gpr-2, or lin-5 largely eliminates these pulling forces (Nguyen-Ngoc et al. 2007), while specific loss of LIN-5 from spindle poles has no effect (van der Voet et al. 2009). Thus, cortical localization of LIN-5, through G $\alpha-$ GPR-1/GPR-2 interaction, is needed for the pulling forces that position the spindle in mitosis.

G $\alpha$-GPR-LIN-5 contributes to cortical pulling forces through the recruitment of a dynein motor complex to the cell periphery (Couwenbergs et al. 2007; NguyenNgoc et al. 2007). Dynein anchored by G $\alpha-$ GPR-LIN-5 attaches microtubule plus ends to the cell cortex, while depolymerization of the microtubules ends is thought to be largely responsible for force generation (Kozlowski et al. 2007; Nguyen-Ngoc et al. 2007; Laan et al. 2012). Myristoylation of the $\mathrm{G} \alpha$ subunit allows membrane attachment of the complex, and, based on the analysis of human NuMA, the N-terminal part of LIN-5/NuMA mediates dynein interaction (Kotak et al. 2012). While these molecular interactions are conserved in the animal kingdom, variations are used in development. In meiosis of the C. elegans female pronucleus, LIN-5 and dynein are needed to rotate the meiotic spindle in order to expel the polar bodies. Instead of G $\alpha-$ GPR-1/GPR-2, a complex of ASPM-1 (abnormal spindle-like, microcephaly associated) and calmodulin anchors LIN-5 and dynein at the spindle poles to mediate this rotation (van der Voet et al. 2009). In planar cell polarity, the Frizzled receptor and Dishevelled effector orient the spindle and division plane. Dishevelled interacts with Mud in Drosophila SOP cells and with NuMA during zebra fish gastrulation to engage the dynein motor complex in this process (Morin and Bellaïche 2011). Thus, the LIN-5/Mud/NuMA coiled-coil protein acts as a general dynein adaptor in spindle positioning. This adaptor also functions as an important target of spatiotemporal regulation of spindle-pulling forces, as discussed in the next section.

\subsection{Regulation of Cortical Force Generation in the C. elegans One-Cell Embryo}

The asymmetric localization of PAR proteins in the $C$. elegans embryo causes the spindle to migrate off-center in mitosis. As compared to Drosophila NBs and SOP cells, the distribution of cortical pulling force proteins is more dynamic and less asymmetric in the $C$. elegans zygote, and a combination of several factors may determine the plane of cell cleavage. Below, we review the mechanisms that have 
been proposed to contribute to asymmetric pulling forces in the one-cell C. elegans embryo.

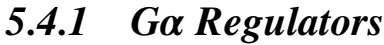

During delamination of the Drosophila NB, the polarity of the neuroepithelium is maintained, and apically localized PAR3-PAR6-aPKC recruits Inscuteable to the apical side of the cell. Pins and Goi accumulate at the same side, presumably recruited by Inscuteable, thus cell polarity corresponds directly to the asymmetry in cortical force generators. By contrast, the GOA-1 and GPA-16 Goi/o subunits show uniform localization at the cortex of the $C$. elegans zygote (Colombo et al. 2003; Gotta et al. 2003; Srinivasan et al. 2003). Nevertheless, the regulation of the active versus inactive state of the heterotrimeric $G$ protein could create asymmetry in pulling forces. The activity of $\mathrm{G}$ proteins depends on the associated guanosine nucleotide, GTP or GDP. As for canonical G-protein signaling, GEF and GAP proteins have been identified that affect GOA-1 and GPA-16 Goi/o activity in spindle positioning. However, GPR-1/GPR-2 associates specifically with Goi/o-GDP, behaves as a GDP-dissociation inhibitor (GDI), and competes with G $\beta \gamma$ in G $\alpha$-GDP binding. While the regulation of the GTP binding and hydrolysis cycle is clearly critical in pulling force generation, it remains puzzling if and how this level of G $\alpha$ regulation contributes to the spatiotemporal control of spindle positioning.

\subsubsection{The Go GTPase Cycle Is Essential for Pulling Force Generation}

An important regulator of $\mathrm{G} \alpha$ in spindle positioning is the "resistant to inhibitors of cholinesterase 8" (RIC-8) protein. The ric-8 gene was identified in a genetic screen for factors that mediate neurotransmitter release, a process regulated by heterotrimeric G-protein signaling (Miller et al. 1996). Remarkably, ric-8 and goa-l showed closely related functions during spindle positioning in the early embryo (Miller and Rand 2000). Insight in the molecular function of RIC-8 came from studies that identified mammalian RIC-8A and RIC-8B as binding partners of Goi/o/q (Tall et al. 2003). Further analysis revealed that RIC-8A exhibits potent GEF activity and associates with the G $\alpha \cdot$ GDP monomer and nucleotide-free transition state, but not with G $\alpha \cdot G T P$ or the trimeric G $\alpha \cdot G D P-G \beta \gamma$ complex. RIC-8 interacts with GOA-1 as well as GPA-16 G $\alpha$ in C. elegans and is a GEF for GOA-1 in vitro. The RIC-8 protein is present uniformly in the cytoplasm and weakly at the cortex. Strong inactivation of ric- 8 , by combined mutation and RNAi, causes loss of pulling forces in the early $C$. elegans embryo and resembles goa-1/gpa-16 double RNAi or knockdown of other components of the LIN-5 complex (Afshar et al. 2004). Thus, RIC- 8 acts as a general positive regulator of G $\alpha$-mediated spindle positioning. 
It appears paradoxical that the RIC-8 GEF and GPR-1/GPR-2 GDI proteins display opposite biochemical activity while both promote spindle-pulling forces. GEF requirement may indicate that the G $\alpha$ subunit needs to go through the GTP binding/hydrolysis cycle in this process. Mammalian RIC-8A does not activate $\mathrm{G} \alpha \cdot \mathrm{GDP}-\mathrm{G} \beta \gamma$ trimers, but catalyzes nucleotide exchange of free G $\alpha \cdot \mathrm{GDP}$ and Go.GDP in complex with a GoLoco/GPR motif protein and NuMA (Tall and Gilman 2005). Thereby, RIC-8 triggers the dissociation of the Go.GDP-LGNNuMA complex. Thus, force generation may involve dissociation of the pulling force complex or generation of G $\alpha$ GTP. A different model came from observations in NBs and SOP cells in Drosophila and of RIC-8A and RIC-8B knockout mouse ES cells (David et al. 2005; Hampoelz et al. 2005; Gabay et al. 2011). These studies showed that RIC-8 is needed for plasma membrane association of newly synthesized G $\alpha$ subunits. Acting as a molecular chaperone, RIC- 8 might use the nucleotide switch to dissociate from the properly folded and ER membrane associated $\mathrm{G} \alpha \cdot \mathrm{GTP}$ molecule. C. elegans GOA-1 and GPA-16 show differential interactions with RIC-8. RIC-8 acts as a GEF for GOA-1 but not GPA-16, and RIC-8 controls the cortical localization and protein level of GPA-16, but not GOA-1 (Afshar et al. 2004, 2005). As ric-8 RNAi resembles the goa-1/gpa-16 double knockdown phenotype, both chaperone and GEF activities of RIC- 8 may contribute to pulling force generation.

The contribution of a GTP binding/hydrolysis cycle in noncanonical G-protein signaling is further supported by the involvement of a possible G $\alpha$ GAP (GTPaseactivating protein). Examining all C. elegans proteins with "regulator of G-protein signaling" (RGS) GTPase activation domains, RGS-7 was recognized for its essential function in embryonic development (Hess et al. 2004). One-cell embryos lacking $\mathrm{rgs}_{\mathrm{s}} \mathrm{7}$ function showed increased movement and rocking of the posterior spindle pole and exaggerated asymmetric division. The severing of the spindle midzone with a UV laser beam demonstrated that the increased posterior displacement of the spindle results from decreased anterior pulling forces in rgs-7 mutant embryos. RGS-7 is present at the cortex, though only detectable from the two-cell stage onward (Hess et al. 2004). It remains unknown why the loss of an apparently uniformly distributed RGS-7 protein reduces only anterior pulling forces. However, genetic and biochemical experiments strongly support that RGS-7 acts as a GAP for GOA-1.

The G $\beta \gamma$ dimer is also an important negative regulator of pulling force generation, as it competes with GPR-1/GPR-2 for G $\alpha$.GDP. As mentioned above, RNAi of gpb- 1 and gpc-2 causes abnormal centrosome movements and spindle orientation. Based on combined RNAi with $\mathrm{G} \alpha$, this phenotype results from hyperactive $\mathrm{G} \alpha$ that is no longer sequestered by $\mathrm{G} \beta \gamma$, and not from loss of $\mathrm{G} \beta \gamma$-specific effector signaling (Tsou et al. 2003). Spindle-severing experiments showed that gpb-1 RNAi increases spindle-pulling forces in the anterior (Afshar et al. 2004, 2005). Thus, the asymmetry in $\mathrm{G} \beta \gamma$ could contribute to the asymmetry in pulling forces and posterior displacement of the spindle in mitosis. A recent study showed dynamic regulation of cortical GPB-1 levels and trafficking through both early endosomes and recycling endosomes (Thyagarajan et al. 2011). In metaphase of the 
zygote, trafficking rates are higher in the anterior than the posterior, and more GPB-1 remains present in endosomal vesicles in the posterior. Thus, a larger fraction of the uniformly distributed $\mathrm{G} \alpha$ protein may be available for GPR-1/ GPR-2 association in the posterior. This provides a potential mechanism for spatiotemporal regulation of spindle positioning.

\subsubsection{GPR-1/GPR-2 Regulators}

Since the discovery of the GPR-1/GPR-2 proteins, asymmetry in their localization has been proposed to be responsible for asymmetric pulling forces (Colombo et al. 2003; Gotta et al. 2003). However, our group did not detect statistically relevant posterior enrichment of GPR-1/GPR-2 (or LIN-5) in metaphase, while spindle-pulling forces are clearly higher in the posterior at that stage (Srinivasan et al. 2003; Galli et al. 2011; Berends et al. 2013). The localization of GPR-1/GPR2 is quite dynamic and likely subjected to regulatory mechanisms. GPR-1/GPR-2 and LIN-5 become anteriorly enriched during polarity establishment and prophase of the first mitotic division, which contributes to pronuclear centration (Tsou et al. 2003; Park and Rose 2008). Subsequently, GPR-1/GPR-2 and LIN-5 redistribute to become higher at the posterior cortex in mitosis. This enrichment is limited but detectable in anaphase; however, it may either follow or cause asymmetry in pulling forces (Colombo et al. 2003; Gotta et al. 2003; Srinivasan et al. 2003; Galli et al. 2011; Berends et al. 2013). Moreover, asymmetry in cortical dynein localization has not been detected during any phase of $C$. elegans zygotic division (Nguyen-Ngoc et al. 2007). Thus, the question whether asymmetric distribution of the pulling force complex is responsible for asymmetric spindle positioning has not been conclusively answered.

The localization of GPR-1/GPR-2 requires not only G $\alpha$ but also LIN-5 (Gotta et al. 2003; Srinivasan et al. 2003). A mechanism proposed for mammalian LGN may explain this dual dependence (Du and Macara 2004). The N-terminal TPR domains of LGN and C-terminal GoLoco/GPR motifs engage in intramolecular interactions that cause a closed protein conformation. The binding of either Goi or NuMA abolishes this intramolecular interaction and allows for simultaneous binding of the other partner (Du and Macara 2004). Structural studies indicate that the LGN GoLoco domains interact in tandem with the LGN TPR repeats (Pan et al. 2013). Given that GPR-1/GPR-2 has only few confirmed TPR and GoLoco/ GPR motifs, it is unclear whether the conformational switch model proposed for LGN should apply to GPR-1/GPR-2 as well. The dependence on both G $\alpha$ and LIN-5 supports that GPR-1/GPR-2 uses a related mechanism for its cortical localization.

An additional regulatory protein might induce asymmetry in GPR-1/GPR-2 function. LET-99 is a DEP (Dishevelled/EGL-10/Pleckstrin) domain protein that antagonizes cortical localization of G $\alpha-$ GPR-1/GPR-2 (Tsou et al. 2003; Bringmann et al. 2007; Park and Rose 2008). The let-99 mutant phenotype 
resembles aspects of the $g p b-1$ RNAi phenotype and indicates negative regulation of cortical G $\alpha-\mathrm{GPR}-1 / \mathrm{GPR}-2$. Both anterior and posterior PAR proteins inhibit LET-99 at the cortex, which restricts LET-99 localization to a cortical band at about $60 \%$ of the long axis of the one-cell embryo. The identification of the LET-99 band has resulted in a cortical force model with three instead of two (anterior and posterior) domains. Negative regulation of force generation in the posterior LET-99 band (possibly in combination with asymmetric distribution of cortical GPR-1/GPR-2) results in net anterior pulling forces during pronuclear centration and net posterior pulling forces from metaphase onwards (Tsou et al. 2003; Couwenbergs et al. 2004; Krueger et al. 2010). This indicates the possibility that not anterior-posterior GPR-1/GPR-2 asymmetry per se, but rather reduced pulling on astral microtubules that reach the LET-99 lateral band, is responsible for a net increase in posterior pulling forces and spindle displacement in mitosis.

An RNAi screen for defects in pronuclear and spindle movements identified casein kinase-1 (CSNK-1) as a pulling force regulator (Panbianco et al. 2008). CSNK-1 is membrane associated and enriched in the anterior of the C. elegans zygote in a PAR-dependent manner. CSNK-1 negatively regulates the localization of GPR-1/GPR-2-LIN-5, possibly indirectly by confining the PIP2-generating PIP2 kinase PPK-1 to the posterior. PPK-1 or PIP2 may positively regulate the localization of GPR-1/GPR-2-LIN-5, although neither GPR-1/GPR-2 nor LIN-5 has a known PIP2-binding domain (Panbianco et al. 2008). The protein phosphatase PPH-6 and its associated subunit Sit4p-associated protein-1 (SAP-1) also promote GPR-1/GPR-2 localization and spindle-pulling forces in anaphase (Afshar et al. 2010). Co-depletion of CSNK-1 and PPH-6 resembles the PPH-6/SAPS-1 depleted phenotype of decreased cortical GPR-1/GPR-2 localization and spindle forces. Thus, CSNK-1 may act on PPH-6 to inhibit GPR-1/GPR-2-LIN-5 localization in the anterior. While the exact molecular mechanisms remain unclear, both kinases and phosphatases contribute to regulation of GPR-1/GPR-2 localization.

\subsubsection{LIN-5 Regulators}

Because G $\alpha-$ GPR-LIN-5 form a trimeric complex, the regulation of $\mathrm{G} \alpha$ and GPR-1/GPR-2 levels described above also applies to cortical localization of LIN-5. In addition, LIN-5 is also subjected to specific controls. Immunopurification followed by mass spectrometry showed extensive phosphorylation of LIN-5 at 25 different residues (Galli et al. 2011). Stable isotope labeling combined with kinase knockdown and quantitative phosphopeptide analysis revealed that four LIN-5 serine residues are phosphorylated directly by the atypical protein kinase $\mathrm{C}$ 3 (PKC-3), in a cyclin-dependent kinase 1 (CDK-1)-dependent manner. PKC-3 is part of the anterior PAR complex, while CDK-1 is a key positive regulator of mitotic entry, thus establishing a direct connection between the LIN-5 complex, PAR polarity, and cell cycle progression. Phosphorylation of LIN-5 by PKC-3 occurs in the anterior, peaks in metaphase, and then disappears rapidly (Galli 
a

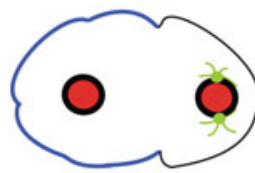

Pronuclear migration

\section{b}

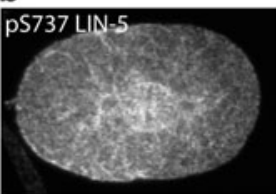

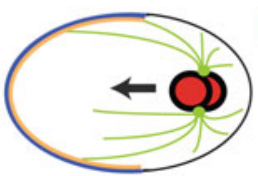

Centration

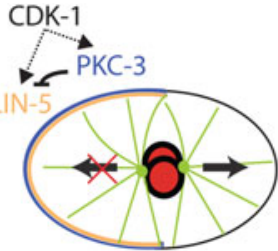

Switch to posterior directed spindle movements

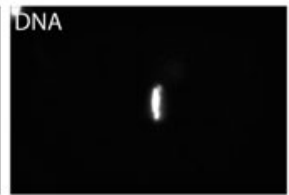

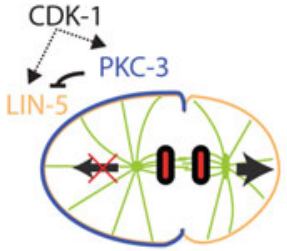

Spindle displacement towards posterior

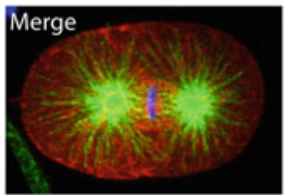

Fig. 5.5 PKC-3 phosphorylates LIN-5 to regulate cortical pulling forces. (a) Different phases of the first mitotic division of the $C$. elegans embryo, illustrating the positioning of the spindle apparatus (green) and localization of PKC-3 (blue) and LIN-5 (yellow). The schemes indicate the interactions between LIN-5, PKC-3, and (possibly) CDK-1. The arrows in the figures signify the direction of pulling forces. (b) Immunohistochemical staining of a one-cell embryo in metaphase, with antibodies recognizing LIN-5 phosphorylated on S737 (red), $\alpha$-tubulin to mark the spindle (green), and DAPI to visualize DNA (blue). pS737 LIN-5 is clearly enriched anteriorly during this mitotic phase. Figure adapted after Galli et al. (2011)

et al. 2011). Spindle midzone severing experiments in combination with non-phosphorylatable and phosphorylation-mimicking LIN-5 mutations demonstrated that the PKC-3-specific phosphorylation of LIN-5 inhibits pulling forces. This spatiotemporal regulation coincides with the switch from anterior-directed movement of the pronuclei-centrosomal complex during centration to posterior migration of the spindle in metaphase/anaphase (Fig. 5.5).

To promote symmetric cell division, apically localized aPKC antagonizes spindle-pulling forces in polarized Drosophila and mammalian epithelial cells (Hao et al. 2010; Guilgur et al. 2012). Madin-Darby canine kidney (MDCK) cells in 3D culture form organized cysts with a single luminal epithelial layer. To instruct a planar spindle position, aPKC phosphorylates LGN/Pins, which creates a 14-3-3 protein binding site and prevents association with Goi (Hao et al. 2010). Thus, phosphorylation of pulling force complex components by aPKC provides a direct way to coordinate the cell division plane with cell polarity in worms, flies, and mammals. Similarly, mitotic kinases are likely to couple cell cycle progression and pulling force generation through phosphorylation of LIN-5/NuMA or GPR/LGN. The LIN-5 phosphorylation by PKC-3, described above, depends indirectly on CDK-1 (Galli et al. 2011). CDK-1 phosphorylation possibly activates PKC-3 or might prime LIN-5 for subsequent phosphorylation by PKC-3. Direct phosphorylation of NuMA by CDK-1 has been implicated in dynein localization in mammalian cells grown in culture (Kiyomitsu and Cheeseman 2013; Kotak et al. 2013). NuMA phosphorylation by CDK-1 during metaphase negatively affects cortical 
dynein levels and is antagonized by the continuously active protein phosphatase PPP2CA. This mechanism explains an observed increase in cortical dynein levels from metaphase to anaphase, following inactivation of CDK-1. Additional phosphorylations by cell cycle and polarity kinases will probably contribute to spatiotemporal control of cortical pulling forces.

\subsubsection{Dynein}

The recruitment of cytoplasmic dynein appears the most important function of the LIN-5 complex. Dynein acts in association with various regulator and adaptor proteins, including the multiple subunit dynactin complex (Raaijmakers et al. 2013). Components of cytoplasmic dynein as well as dynactin are conserved between $C$. elegans and mammals and include potential adaptors for LIN-5 association as well as other regulators of localization and activity (O'Rourke et al. 2007; Raaijmakers et al. 2013). For example, the dynein adaptor lissencephaly 1 (LIS-1) is required for cortical pulling forces (Nguyen-Ngoc et al. 2007). In vitro studies have shown that LIS-1 promotes dynein's continued association with microtubules (Huang et al. 2012). Continued attachment to depolymerizing microtubule ends is likely needed for pulling force generation.

Dynein acting independently of the LIN-5 pulling force complex has been proposed to contribute to centration movements (Kimura and Kimura 2011). The dynein light chain protein, dynein roadblock (DYRB-1), anchors organelles for transport along microtubules. Because of the resistance of the viscous cytoplasm, this generates a dragging force at centrosomes. This mechanism may contribute to centration because microtubules extending toward the anterior are longer, and the majority of organelles are anterior of the maternal and paternal pronucleus at the time of meeting (Kimura and Kimura 2011). Another mechanism proposed to contribute to the centration of the pronuclei-centrosomal complex is the sliding of microtubules along the cortex, when microtubules are not attached end-on but laterally by a cortical LIN-5/dynein complex (Gusnowski and Srayko 2011). Lateral sliding is more prominent during centration than during anaphase and also more evident in the anterior than the posterior part of the embryo. It is unclear whether this sliding is actively regulated or a consequence of the geometry of the spindle and angle under which microtubules reach the cortex. Pushing forces of microtubules that buckle up against the cortex without being captured by dynein may further promote centration of the pronuclei-centrosomal complex (Laan et al. 2012). 


\subsubsection{Microtubule Dynamics}

The generation of cortical pulling forces depends strongly on the dynamic instability of microtubules. This is supported by the finding that pulling force generation is lost after the administration of microtubule-stabilizing drugs (Nguyen-Ngoc et al. 2007). Microtubule plus tips grow with an estimated speed of $\sim 0.5 \mu \mathrm{m} / \mathrm{s}$ and depolymerize with a speed of $\sim 0.84 \mu \mathrm{m} / \mathrm{s}$ during catastrophe (Kozlowski et al. 2007). Live observations of embryos expressing plus-end tracking EBP-2:: GFP have shown that catastrophe rarely occurs before a growing microtubule reaches the cortex. When reaching the cortex, however, catastrophe follows within 1-2 s (Kozlowski et al. 2007). Microtubules often reach the cortex as bundles of multiple filaments, which can be captured by pulling force complexes. In vitro experiments have indicated that a single depolymerizing microtubule generates six to ten times as much force as a motor protein $(\sim 50 \mathrm{pN}$, a single dynein motor $\sim 7$ to 8 pN) (Grishchuk et al. 2005; Kozlowski et al. 2007). It is possible that dynein motor activity is only required to maintain contact with the depolymerizing microtubule (Laan et al. 2012).

Force generators that are anchored in a more rigid cortex are less able to maintain contact with the depolymerizing microtubule and thus produce less force (Kozlowski et al. 2007). So far, there is no experimental evidence showing an asymmetry in spindle geometry between the anterior and posterior part of the $C$. elegans zygote. However, F-actin is enriched at the anterior cortex when the zygote enters mitosis. This most likely increases cortical rigidity and must therefore decrease the forces generated by anterior pulling force complexes. Indeed, several groups observed substantially increased pulling forces in the anterior after actin depletion by drug treatment (Afshar et al. 2010; Redemann et al. 2010; Berends et al. 2013). Thus, in addition to the regulation of the pulling force complex, a difference in the cortical rigidity caused by actin accumulation provides a possible cause of pulling force asymmetry.

\subsection{Concluding Remarks}

Through controlled spindle positioning, polarized cells decide between symmetric and asymmetric cell division and create daughter cells of the correct sizes at the appropriate positions. Studies in worms, flies, and mammals have provided detailed descriptions of representative symmetric and asymmetric cell divisions. By now, most of the basic players in polarity establishment and spindle positioning may be identified. It remains incompletely understood, however, how cell and tissue polarity translates to the proper positioning of the mitotic spindle. While the one-cell C. elegans embryo offers a relatively simple and well-tractable model, providing a complete answer to this question has proven to be remarkably difficult. Polaritydependent differences in pulling force component localization, phosphorylation, 
antagonist association, and actin accumulation have all been described to contribute to the lower anterior compared to posterior pulling forces that position the spindle during asymmetric division of the $C$. elegans zygote. Importantly, the identified principles appear to be conserved and apply broadly to the regulation of the cell division plane in other cell types and organisms.

\section{References}

Afshar K, Werner ME, Tse YC, Glotzer M, Gönczy P (2010) Regulation of cortical contractility and spindle positioning by the protein phosphatase $6 \mathrm{PPH}-6$ in one-cell stage $\mathrm{C}$. elegans embryos. Development 137:237-247

Afshar K, Willard FS, Colombo K, Johnston CA, Mccudden CR, Siderovski DP, Go P, Hill C, Carolina N (2004) RIC-8 is required for GPR-1/2-dependent Ga function during asymmetric division of C. elegans embryos The University of North Carolina at Chapel Hill. Cell 119:219-230

Afshar K, Willard FS, Colombo K, Siderovski DP, Gönczy P (2005) Cortical localization of the Galpha protein GPA-16 requires RIC-8 function during C. elegans asymmetric cell division. Development 132:4449-4459

Albertson G (1978) Cell cycling and DNA replication in a mutant blocked in cell division in the nematode Caenorhabditis elegans. Dev Biol 178:165-178

Bei Y, Hogan J, Berkowitz LA, Soto M, Rocheleau CE, Pang KM, Collins J, Mello CC (2002) SRC-1 and Wnt signaling act together to specify endoderm and to control cleavage orientation in early C. elegans embryos. Dev. Cell 3:113-125

Bellaïche Y, Radovic A, Woods DF, Hough CD, Parmentier ML, O'Kane CJ, Bryant PJ, Schweisguth F (2001) The Partner of Inscuteable/Discs-large complex is required to establish planar polarity during asymmetric cell division in Drosophila. Cell 106:355-366

Berends CWH, Munoz J, Portegijs V, Schmidt R, Grigoriev I, Boxem M, Akhmanova A, Heck AJR, van den Heuvel S (2013) F-actin asymmetry and the ER-associated TCC-1 protein contribute to stereotypic spindle movements in the C. elegans embryo. Mol Biol Cell 24:2201-2215

Bergmann DC, Lee M, Robertson B, Meng-Fu B, Rose LS, Wood WB (2003) Bergmannembryonic handedness choice in C. elegans involves the Go protein GPA-16.pdf. Development 130:5731-5740

Bowman SK, Neumüller RA, Novatchkova M, Du Q, Knoblich JA (2006) The Drosophila NuMA Homolog Mud regulates spindle orientation in asymmetric cell division. Dev Cell 10:731-742

Bringmann H, Cowan CR, Kong J, Hyman AA (2007) LET-99, GOA-1/GPA-16, and GPR-1/2 are required for aster-positioned cytokinesis. Curr Biol 17:185-191

Cabernard C, Prehoda KE, Doe CQ (2010) A spindle-independent cleavage furrow positioning pathway. Nature 467:91-94

Colombo K, Grill SW, Kimple RJ, Willard FS, Siderovski DP, Gönczy P (2003) Translation of polarity cues into asymmetric spindle positioning in Caenorhabditis elegans embryos. Science 300:1957-1961

Couwenbergs C, Labbé J-C, Goulding M, Marty T, Bowerman B, Gotta M (2007) Heterotrimeric $\mathrm{G}$ protein signaling functions with dynein to promote spindle positioning in C. elegans. Cell Biol 179:15-22

Couwenbergs C, Spilker ACA, Gotta M (2004) Control of Embryonic Spindle Positioning and Gî \pm Activity by C. elegans RIC-8. Curr Biol 14:1871-1876

Cowan CR, Hyman AA (2007) Acto-myosin reorganization and PAR polarity in C. elegans. Development 134:1035-1043 
David NB, Martin CA, Segalen M, Rosenfeld F, Schweisguth F, Bellaïche Y (2005) Drosophila Ric-8 regulates Galphai cortical localization to promote Galphai-dependent planar orientation of the mitotic spindle during asymmetric cell division. Nat Cell Biol 7:1083-1090

Du Q, Macara IG (2004) Mammalian Pins is a conformational switch that links NuMA to heterotrimeric G proteins. Cell 119:503-516

Fietz SA, Huttner WB (2011) Cortical progenitor expansion, self-renewal and neurogenesis-a polarized perspective. Curr Opin Neurobiol 21:23-35

Gabay M, Pinter ME, Wright FA, Chan P, Murphy AJ, Valenzuela DM, Yancopoulos GD, Tall GG (2011) Ric-8 proteins are molecular chaperones that direct nascent $\mathrm{G}$ protein $\alpha$ subunit membrane association. Sci Signal 4:ra79

Galli M, van den Heuvel S (2008) Determination of the cleavage plane in early C. elegans embryos. Annu Rev Genet 42:389-411

Galli M, Muñoz J, Portegijs V, Boxem M, Grill SW, Heck AJR, van den Heuvel S (2011) aPKC phosphorylates NuMA-related LIN-5 to position the mitotic spindle during asymmetric division. Nat Cell Biol 13:1132-1138

Gotta M, Ahringer J (2001) Distinct roles for Galpha and Gbetagamma in regulating spindle position and orientation in Caenorhabditis elegans embryos. Nat Cell Biol 3:297-300

Gotta M, Dong Y, Peterson YYK, Lanier SSM, Ahringer J (2003) Asymmetrically Distributed C. elegans Homologs of AGS3/PINS Control Spindle Position in the Early Embryo. Curr Biol 13:1029-1037

Knoblich JA (2008) Mechanisms of asymmetric stem cell division. Cell 132:583-597

Grishchuk EL, Molodtsov MI, Ataullakhanov FI, McIntosh JR (2005) Force production by disassembling microtubules. Nature 438:384-388

Guilgur LG, Prudêncio P, Ferreira T, Pimenta-Marques AR, Martinho RG (2012) Drosophila aPKC is required for mitotic spindle orientation during symmetric division of epithelial cells. Development 139:503-513

Gusnowski EM, Srayko M (2011) Visualization of dynein-dependent microtubule gliding at the cell cortex: implications for spindle positioning. J Cell Biol 194:377-386

Hampoelz B, Hoeller O, Bowman SK, Dunican D, Knoblich JA (2005) Drosophila Ric-8 is essential for plasma-membrane localization of heterotrimeric $G$ proteins. Nat Cell Biol 7:1099-1105

Hao Y, Du Q, Chen X, Zheng Z, Balsbaugh JL, Maitra S, Shabanowitz J, Hunt DF, Macara IG (2010) Par3 controls epithelial spindle orientation by aPKC-mediated phosphorylation of apical Pins. Curr Biol 20:1809-1818

Hess HA, Röper J-C, Grill SW, Koelle MR (2004) RGS-7 completes a receptor-independent heterotrimeric $\mathrm{G}$ protein cycle to asymmetrically regulate mitotic spindle positioning in C. elegans. Cell 119:209-218

Hoege C, Hyman AA (2013) Principles of PAR polarity in Caenorhabditis elegans embryos. Nat Rev Mol Cell Biol 14:315-322

Horvitz HR, Herskowitz I (1992) Mechanisms of asymmetric cell division: two Bs or not two Bs, that is the question. Cell 68:237-255

Huang J, Roberts AJ, Leschziner AE, Reck-Peterson SL (2012) Lis1 acts as a "clutch" between the ATPase and microtubule-binding domains of the dynein motor. Cell 150:975-986

Izumi Y, Ohta N, Itoh-Furuya A, Fuse N, Matsuzaki F (2004) Differential functions of G protein and Baz-aPKC signaling pathways in Drosophila neuroblast asymmetric division. J Cell Biol 164:729-738

Kaltschmidt JA, Davidson CM, Brown NH, Brand AH (2000) Rotation and asymmetry of the mitotic spindle direct asymmetric cell division in the developing central nervous system. Nat Cell Biol 2:7-12

Kemphues KJ, Priess JR, Morton DG, Cheng NS (1988) Identification of genes required for cytoplasmic localization in early C. elegans embryos. Cell 52:311-320

Kimple RJ, Kimple ME, Betts L, Sondek J, Siderovski DP (2002) Structural determinants for GoLoco-induced inhibition of nucleotide release by Galpha subunits. Nature 416:878-881 
Kimura K, Kimura A (2011) Intracellular organelles mediate cytoplasmic pulling force for centrosome centration in the Caenorhabditis elegans early embryo. Proc Natl Acad Sci U S A 108:137-142

Kiyomitsu T, Cheeseman IM (2013) Cortical dynein and asymmetric membrane elongation coordinately position the spindle in anaphase. Cell 154:391-402

Knoblich JA (2008) Mechanisms of asymmetric stem cell division. Cell 132:583-597

Knoblich JA (2010) Asymmetric cell division: recent developments and their implications for tumour biology. Nat Rev Mol Cell Biol 11:849-860

Kotak S, Busso C, Gönczy P (2012) Cortical dynein is critical for proper spindle positioning in human cells. J Cell Biol 199:97-110

Kotak S, Busso C, Gönczy P (2013) NuMA phosphorylation by CDK1 couples mitotic progression with cortical dynein function. EMBO J 32:2517-2529

Kozlowski C, Srayko M, Nedelec F (2007) Cortical microtubule contacts position the spindle in C. elegans embryos. Cell 129:499-510

Krueger LE, Wu J-C, Tsou M-FB, Rose LS (2010) LET-99 inhibits lateral posterior pulling forces during asymmetric spindle elongation in C. elegans embryos. J Cell Biol 189:481-495

Laan L, Pavin N, Husson J, Romet-Lemonne G, van Duijn M, López MP, Vale RD, Jülicher F, Reck-Peterson SL, Dogterom M (2012) Cortical dynein controls microtubule dynamics to generate pulling forces that position microtubule asters. Cell 148:502-514

Lechler T, Fuchs E (2005) Asymmetric cell divisions promote stratification and differentiation of mammalian skin. Nature 437:275-280

Lorson MA, Horvitz HR, van den Heuvel S (2000) LIN-5 is a novel component of the spindle apparatus required for chromosome segregation and cleavage plane specification in Caenorhabditis elegans. J Cell Biol 148:73-86

Martin-mccaffrey L, Willard FS, Pajak A, Dagnino L, Souza SJAD (2005) RGS14 is a microtubule-associated protein. Cell Cycle 4:953-960

Miller KG, Alfonso A, Nguyen M, Crowell JA, Johnson CD, Rand JB (1996) A genetic selection for Caenorhabditis elegans synaptic transmission mutants. Proc Natl Acad Sci U S A 93:12593-12598

Miller KG, Rand JB (2000) A role for RIC-8 and GOA-1 in regulating a subset of centrosome movements during early embryogenesis in Caenorhabditis elegans. Genetics 8:1649-1660

Morin X, Bellaïche Y (2011) Mitotic spindle orientation in asymmetric and symmetric cell divisions during animal development. Dev Cell 21:102-119

Nguyen-Ngoc T, Afshar K, Gönczy P (2007) Coupling of cortical dynein and G alpha proteins mediates spindle positioning in Caenorhabditis elegans. Nat Cell Biol 9:1294-1302

O'Rourke SM, Dorfman MD, Carter JC, Bowerman B (2007) Dynein modifiers in C. elegans: light chains suppress conditional heavy chain mutants. PLoS Genet 3:e128

Pan Z, Zhu J, Shang Y, Wei Z, Jia M, Xia C, Wen W, Wang W, Zhang M (2013) An autoinhibited conformation of LGN reveals a distinct interaction mode between GoLoco Motifs and TPR motifs. Structure 21:1007-1017

Panbianco C, Weinkove D, Zanin E, Jones D, Divecha N, Gotta M, Ahringer J (2008) A casein kinase 1 and PAR proteins regulate asymmetry of a PIP(2) synthesis enzyme for asymmetric spindle positioning. Dev Cell 15:198-208

Park DH, Rose LS (2008) Dynamic localization of LIN-5 and GPR-1/2 to cortical force generation domains during spindle positioning. Dev Biol 315:42-54

Raaijmakers JA, Tanenbaum ME, Medema RH (2013) Systematic dissection of dynein regulators in mitosis. J Cell Biol 201:201-215

Redemann S, Pecreaux J, Goehring NW, Khairy K, Stelzer EHK, Hyman AA, Howard J (2010) Membrane invaginations reveal cortical sites that pull on mitotic spindles in one-cell $\mathrm{C}$ elegans embryos PLoS One 5:e12301

Schaefer M, Petronczki M, Dorner D, Forte M, Knoblich JA (2001) Heterotrimeric G proteins direct two modes of asymmetric cell division in the Drosophila nervous system. Cell 107:183-194 
Schaefer M, Shevchenko A, Knoblich JA (2000) A protein complex containing Inscuteable and the Galpha-binding protein Pins orients asymmetric cell divisions in Drosophila. Curr Biol 10:353-362

Siller KH, Doe CQ (2009) Spindle orientation during asymmetric cell division. Nat Cell Biol 11:365-374

Srinivasan DDGD, Fisk RMR, Xu H (2003) A complex of LIN-5 and GPR proteins regulates G protein signaling and spindle function in C. elegans. Genes Dev 17:1225-1239

Sulston JE, Horvitz HR (1981) Abnormal cell lineages in mutants of the nematode Caenorhabditis elegans. Dev Biol 82:41-55

Tall GG, Gilman AG (2005) Resistance to inhibitors of cholinesterase 8A catalyzes release of Gai-GTP and nuclear mitotic apparatus protein (NuMA) from NuMA/LGN/Gai-GDP complexes. Proc Natl Acad Sci U S A 102:16574-16589

Tall GG, Krumins AM, Gilman AG (2003) Mammalian Ric-8A (synembryn) is a heterotrimeric Galpha protein guanine nucleotide exchange factor. J Biol Chem 278:8356-8362

Thyagarajan K, Afshar K, Gönczy P (2011) Polarity mediates asymmetric trafficking of the Gbeta heterotrimeric G-protein subunit GPB-1 in C. elegans embryos. Development 138:2773-2782

Tsou M-FB, Hayashi A, Rose LS (2003) LET-99 opposes Galpha/GPR signaling to generate asymmetry for spindle positioning in response to PAR and MES-1/SRC-1 signaling. Development 130:5717-5730

Van der Voet M, Berends CWH, Perreault A, Nguyen-Ngoc T, Gönczy P, Vidal M, Boxem M, van den Heuvel S (2009) NuMA-related LIN-5, ASPM-1, calmodulin and dynein promote meiotic spindle rotation independently of cortical LIN-5/GPR/Galpha. Nat Cell Biol 11:269-277

Williams SE, Beronja S, Pasolli HA, Fuchs E (2011) Asymmetric cell divisions promote Notchdependent epidermal differentiation. Nature 470:353-358

Zwaal RR, Ahringer J, van Luenen HG, Rushforth A, Anderson P, Plasterk RH (1996) G proteins are required for spatial orientation of early cell cleavages in C. elegans embryos. Cell 86:619-629 